\title{
Graphical vs. Spatial Models of Distributional Semantics
}

\author{
Shufan Mao ${ }^{1}$ and Jon A. Willits ${ }^{1}$ \\ ${ }^{1}$ University of Illinois at Urbana-Champaign
}

\begin{abstract}
Semantic space models based on distributional information and semantic network (graphical) models are two of the most popular models of semantic representation. Both types of models succeed at modeling or explaining various tasks. Both types of models also have limitations. Spatial models have difficulties representing indirect semantic relations, while graphical models have lacked a theoretical account for the construction of their semantic network. In this article, we develop the Distributional Graph Model. The new model resembles semantic space models in the way that it is a representation of semantic memory obtained from statistical learning on a linguistic corpus. But like other graphical models, it is able to capture indirect semantic relatedness as well. Using an artificial language specifically designed to test different types of syntagmatic and paradigmatic relationships, we show that the Distributional Graph Model demonstrates the benefits of both graphical and spatial distributional models.
\end{abstract}

Keywords: semantic memory, distributional semantic models; graphical models; spreading activation

\section{Introduction}

\subsection{Spatial Distributional Models}

Distributional semantic models have become a very popular approach for representing words, concepts, and semantic memory, both in cognitive science and in industry applications. Traditionally, these models encode the meaning of a word as some function of the "distribution" of other words with which that word co-occurs (Landauer \& Dumais, 1997). The models transform linguistic distributional information into a feature space, representing the words as coordinates in the space generated by the words' pattern of co-occurrence with other words. Because words with similar meanings tend to be used in similar ways, the proximity of words within this space ends up being a fairly good representation of word meaning. It is important to point out that most distributional models are properly thought of as "spatial" models, because they are evaluated in a way such that the similarity between words, or the relationships between words, is measured as the similarity between two words' vector representations (Burgess \& K.Lund, 2000). Distributional semantic models have been successful in various semantic tasks. They have been used to model human categorization, semantic priming, word association, and sentence processing, among other tasks see (M. Jones, Willits, \& Dennis, 2014) for a review. 
There are, however, a number of problems with spatial distributional semantic models. One problem is the explanation of different kinds of semantic relationships. Spatial distributional models have a single measure of relatedness: some computation of similarity within the space. Is this one measure of similarity to be used to explain all the different ways that two words can be related? For example, in the verb phrase "chase rabbit", the semantic relation between chase and rabbit is sometimes called syntagmatic, which means they co-occur in the context or bear some kind of thematic relationship (Asher \& Simpson, 1993). In contrast, the relation between rabbit and deer is paradigmatic: the two words are of the same taxonomic category and can be replaced by each other in many contexts (Hjørland, 2015). If similarity in the semantic space is the only way of evaluating semantic relationships, then the model cannot, by definition, be good at representing both syntagmatic and paradigmatic relations in the same semantic space. The better the model gets at representing one, the worse it must do at the other. The more of a word's nearest neighbors are one type of relation, the fewer can be of the other type.

A second problem (which is a downstream consequence of the first), has to do with the ability of spatial models to capture the behavioral effects of mediated or indirect semantic relations. For example, some word pairs, like "zebra" and "roar" may share no direct syntagmatic or paradigmatic relationship. However, they may be indirectly connectable through an intermediary word (like "lion") that shares a paradigmatic relationship with one of the words, and a syntagmatic relationship with the other. Words sharing mediated semantic relationship like zebra and roar tend to be more similar than unrelated words in spatial distributional models, but to date many of these models have done a poor job modeling behavioral responses (like semantic priming) to these relationships, though see (M. N. Jones, Kintsch, \& Mewhort, 2006).

\subsection{Graphical Models}

An alternative to a spatial model of semantic knowledge is a graphical model, where words or concepts are represented in the form of a connected network, instead of as vectors representing points in a semantic space. Notable examples of graphical models of semantics include "Spreading Activation" models (Anderson, 1983; Collins \& Loftus, 1975). In graphical models, concepts are denoted by nodes, and the nodes are connected according to some theory of the kinds of relations that matter to the cognitive system (such as associative strength, or predicative expressions like IS A, HAS, and CAN). In contrast to spatial models, graphical models have no problem representing multiple kinds of relations (because links can be labeled by representation type). This helps with the problem of indirect relations as well. Since graphical structures represent relations between words by connecting the word nodes using edges, it generically forms the indirect relations by intermediate edges and nodes.

However, there are some limitations for the graphical models as well. One critical problem is the question of where the links between the concepts come from? Whether the links in the network are conceptualized as associative strengths or predicative connections (IS A, HAS A, etc.), there are many questions about the formation and structure of the network that cannot be answered without a good theory of where the connections came from in the first place. 


\subsection{Graphical Distributional Model}

One answer to the question of where the links in a semantic graph come from is that they could come from a distributional learning mechanism like those proposed for spatial distributional models. Rotaru, Vigliocco, and Frank (Rotaru, Vigliocco, \& Frank, 2018) presented a graphical model that used distributional similarity to define the connection strength between nodes. They also proposed a spreading activation mechanism to model lexical retrieval, and showed that this model outperformed distributional spatial models without a graphical structure or spreading activation mechanism at predicting accuracy and reaction times for various semantic processing tasks.

Rotaru et al.'s model was an empirical success, but they did not provide a theoretical explanation for why the graphical model with spreading activation outperformed spatial models. In this paper, we address this question, and investigate and compare spatial and graphical distributional models on a new, specially constructed artificial corpus. This corpus was designed to explore what kinds of relationships distributional models can easily learn and represent, and what kinds of relationships that give them difficulty. In the following sections, we will first explain the linguistic corpus on which these models are trained. Then we will explain how we instantiated our spatial and graphical distributional models, how semantic relatedness is measured within these models, and finally the experiments used to compare the models.

\section{Spatial vs. Graphical Experiment}

\subsection{Artificial World and Corpus}

The general goal of this work was to obtain a deeper understanding of the difference in performance between spatial and graphical models. In order to do so, we chose to design an artificial corpus where we could carefully control all relevant factors, such as word frequency, syntactic structure and complexity, and most importantly, the semantic relationships between the words.

We wanted the words' semantic relationships to reflect a real world, consisting of event-based relationships. To do this, we created a very simple artificial world with human agents that have goals, and who pursue those goals through a sequence of actions. Each action was converted into a simple sentence using a generative grammar, and the resulting set of sentences made up corpus on which the models were trained. The general question, then, was how well did the models recover the semantic and syntactic regularities that were used to generate the world?

The full details of the creation of the artificial world and the sentences describing it cannot be exhaustively described due to space limitations. But a brief summary follows. The world was designed to simulate a simple hunter-gather situation. The world consists of human and animal agents in physical locations, as well as selected other objects like tents, fruit trees, and other inanimate objects. Human agents had three drives: hunger, thirst, and sleepiness, which gradually increased over time. At each point in time, each agent selected a goal designed to reduce whichever drive was the strongest (i.e. "GET FOOD", "GET DRINK", or "SLEEP". The selection of a goal leads to the selection of an action plan to achieve that goal. If SLEEP is selected, the agent goes through the actions 'lay down', 'sleep', 'wake up', 'get up'. If 'GET DRINK' is selected, the agent selects the closest source of a drink in the artificial world and then goes through the action sequence 'go to', 'gather', ' boil', etc. If 'GET FOOD' 
is selected, the agent selects the closest food source, and then selects from the possible action sequences that lead to the acquisition of that food object.

In each artificial world, there was one agent, which each took goal-directed actions for a combined total of 10,000 turns. Each action was converted into a simple active sentence using the grammar "AGENT VERB PATIENT." for transitive actions, and "AGENT VERB." for intransitive actions. A snapshot of a resulting corpus is shown in Table 1.

Table 1: Sample sentences from two artificial corpora generated using event-driven semantic generation procedure.

\begin{tabular}{cc}
\hline Corpus 1 & Corpus 2 \\
\hline Andrew go to water & Emily gather beer \\
Andrew gather water & Emily drink beer \\
Andrew boil water & Emily lay down \\
Andrew lay down & Emily wake up \\
Andrew sleep & Emily get up \\
Andrew search & Emily gather deer \\
Andrew go to rabbit & Emily chase deer \\
Andrew trap rabbit & Emily shoot deer \\
Andrew catch rabbit & Emily wash deer \\
Andrew gather rabbit & Emily gather juice \\
\hline
\end{tabular}

By manipulating the frequency of certain verb pair with certain patient, we make some direct syntagmatic relations strong while others weak. Also, by letting certain patients coupling with the same set of verbs (i.e. animals only pairing with hunting and eating verbs while beverages only pairing with certain drinking verbs) we form strong paradigmatic relations.

\subsection{Computational Model}

We constructed two models, a spatial and a graphical model, both built using distributional statistics.

The Spatial Distributional Model (SDM) we tested was similar to the HAL model (Lund \& Burgess, 1996). In our spatial model ${ }^{1}$, we constructed a normalized word-byword co-occurrence matrix by counting forward and backward word co-occurrences within an 9-word window. This matrix was then normalized into the corresponding PPMI matrix. In the resulting matrix, each word's meaning was represented as a vector of normalized co-occurrence values, and semantic relatedness was calculated by computing the cosine similarity of their vectors.

The Distributional Graph Model (DGM) model starts with a co-occurrence matrix representation which is similar to the one from the spatial model, with the difference that the co-occurrence only counts for word pairs which are next to each other within a sentence. And then we convert the matrix into a graphical representation. Each word is represented as a node within the graph, and the nodes were connected if the words

\footnotetext{
${ }^{1}$ We tested a range of HAL-style models varying window size, normalization method, use of SVD, and other parameters. Due to space limitations, we are only reporting the performance of the highest-performing spatial model here.
} 
had a positive value in the Spatial Model's normalized co-occurrence matrix, with the strength of this connection being the normalized co-occurrence value. An example graphical representation is shown in Figure 1.

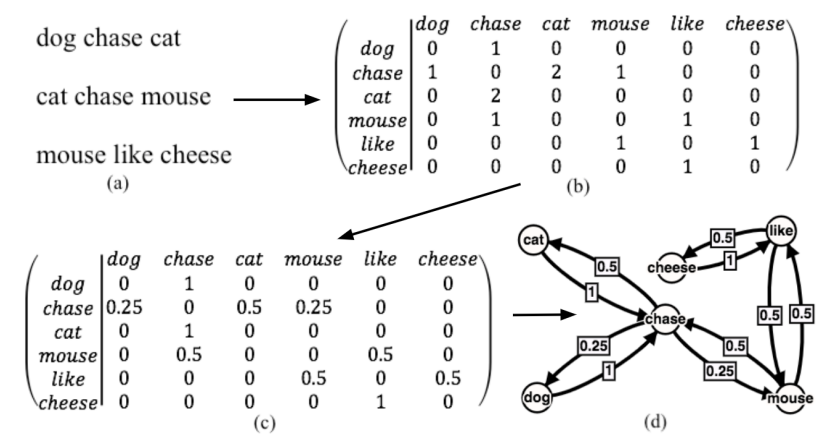

Figure 1: Example graphical model derived from sample corpus (a). Each row of co-occurrence matrix (b) shows a each word's bi-directional, immediately adjacent cooccurrences. This matrix is normalized (c), and nonzero values used to create weighted edges between nodes $(\mathrm{d})$.

In our graphical model, we define the semantic relatedness from word A (source) to word B (target), with notation 'Re(A,B)', in the following way. First, only activate word A with an activation strength of 1.00. All other nodes start with an activation of 0.00. For each time step, the word's activation is divided amongst all a word's connected edges, and proportionally to the edge's strength. The sum of these values determines each word's activation strength at each successive time step.

An example is shown in Figure 2, showing the spread of activation from chase to mouse. At time 0, node 'chase' is activated with activation 1. At time 1, it spreads all its activation to its neighbors, and the amount of activation spreading to the neighbors is proportional to the weights of the edges pointing to them. In this case, 'chase' has 3 neighbors, 'cat', 'dog' and 'mouse', and the weight of the edges pointing to them are $0.5,0.25,0.25$. Therefore, there will be 0.25 activation spreading to 'mouse' at time 1. Since this is the first time that 'mouse' gets activation from chase, we say that the semantic relatedness from 'chase' to 'mouse' in this network is 0.25 . 


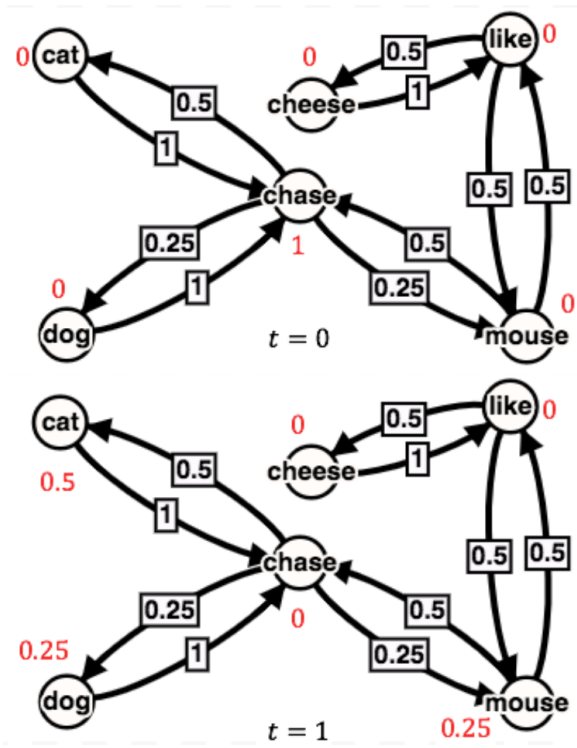

Figure 2: A depiction of the spreading activation process from chase to mouse across two time steps.

This measure of semantic relatedness takes account of both geodesic distance and normalized co-occurrence frequency. If two nodes are closer to each other in the network, there will be less activation flowing elsewhere on the path, so that it is more likely that the target node receives more activation from the source node. On the other hand, if two nodes are connected by some strong connections, then on each step alongside the pathway, it prevents activation from diffusing, so that the target end up with more activation from the source.

With the semantic relatedness measure defined above, we will develop experiments to compare the spatial and graphical models' abilities to bind syntagmatic and paradigmatic relatedness in order to form indirect semantic relations.

\subsection{Experiment Design}

Fifty randomized worlds were created using the procedure described above, and used to generate a corpus of around 2500 (mean=2483, std=131) sentences. Each corpus resulted in a semi-random set of sentences, due to the agent's different randomly initialized drive values, and different starting positions of all the entities. The 50 different corpora were then used to train 50 spatial and 50 graphical models, which were tested to see how well they had learned the underlying semantic rules that had generated the corpus.

\subsubsection{Paradigmatic Relatedness}

To test the models' abilities to capture paradigmatic relations, we compared the models estimation of the relatedness of the items within and between each semantic category (where semantic category was defined in terms of the world's generative rules, with animals, fruits, and drinks as the testable semantic categories). For each model we 
computed the pairwise semantic relatedness (cosine similarity for spatial models, and activation-spreading measure for the graphical model) for all noun-noun pairs from these three categories. Next, we collapsed the relatedness of the noun pairs into a 'within category' group if the two nouns are of the same category, and into a 'between category' otherwise. Finally, we computed the average of the within- and betweencategory groups. If the model learned paradigmatic relatedness, there should be a difference between the within- and between-category average similarity scores.

\subsubsection{Syntagmatic Relatedness}

To test the models' abilities to capture syntagmatic relations, we used a comparison designed to test the models' abilities to pick up on indirect associations that were reasonable for the model to make, but that are not actually possible within the corpus. For example, there are some phrases like "...trap rabbit" and "...shoot deer", where the verb occurs exclusively with a specific direct object (i.e. trapping is only something done to a rabbit, and shooting is only something done to a deer). Beyond these specific sentences, however, the nouns are of strong paradigmatic relations, in that they both occur in many other sentences (e.g. deer and rabbit in hunting sentences, water and juice in drinking sentences, etc.). The question, then, is what the models think of the possibility of sentences like "AGENT shoots rabbit" and "AGENT traps deer", both of which cannot occur in the corpus (due to the rules that generated the world), but both of which seem like reasonable indirect syntagmatic inferences to make. the "shoots/traps deer/rabbit" example can be contrasted with the patient noun water, which should be a much weaker paradigmatic relation to deer and rabbit because it occurs in very few of the same contexts as those words. The question, then, is how the models rank the appropriateness of pairs like 1) "trap rabbit" (legal and frequent in the corpus), 2) "trap deer" (illegal and nonexistent in the corpus, but nonetheless a sensible induction to make), and 3) "trap water" (illegal, nonexistent, and not sensible given low syntagmatic overlap).

Our formal approach comparing the models' abilities mirrors the logic in the example above. For each verb, we generated a "gold standard" key rank-ordering of the plausibility of the semantic relatedness of each noun to that verb, based on the rules used to generate the events in the artificial world. We generated this key matrix in the following way. First, we formed the co-occurrence matrix for verb-noun pairs, where the rows are the nouns, and the columns verbs, and the entries of the matrix are the number of times of noun-verb pair co-occurred in a phrase in the corpus. For each verb, it's highest ranked nouns were the nouns that co-occurred with that verb, in order of co-occurrence. Next, for all nouns which did not co-occur with that verb, we computed their average cosine similarity to all the nouns which did co-occur with the verb. These non-co-occurring nouns were next in the rankings, in terms of their similarity to the nouns that did co-occur with the verb.

The result was that nouns that co-occur with verbs should rank highest in the relatedness to the verb (since the syntagmatic relation is directed by the co-occurrence), while for the non-co-occurring nouns, the more similar they are to the co-occurring nouns (i.e. higher paradigmatic similarity to the nouns that do co-occur), the higher they are in the ranking. Since the standard ranking is obtained by manually binding the syntagmatic relations between co-occur verb-noun pairs (trap and rabbit), and the paradigmatic relations between co-occur and non co-occur nouns (rabbit and deer, water), it is set up as an answer key of the ranking of syntagmatic relatedness for model evaluation. 
For each semantic model, we calculated the semantic relatedness between the verbnoun pairs, e.g. the relatedness between 'trap' and 'rabbit', and used these similarities to compute a rank-ordering for each model. For the spatial models, we took the cosine similarities between the word vectors to get the relatedness score. For the graphical model, we computed the relatedness score for the verb-noun pairs using the spreadingactivation measure described in the earlier section ${ }^{2}$.

We then formed similarity-based rank-orderings for both of the models, and compared these model-based rank orderings to the key ranking (See Table 2).

Table 2: Example of Rankings from Generative Rules (Key), and from graphical and spatial models built from the generated corpora

\begin{tabular}{llll}
\hline Item & Key & Graphical & Spatial \\
\hline (trap,rabbit) & 1 & 1 & 1 \\
(trap,apple) & 2 & 2 & 3 \\
(trap,water) & 3 & 3 & 2 \\
\hline (pour,juice) & 1 & 1 & 2 \\
(pour,peach) & 2 & 2 & 1 \\
(pour,deer) & 3 & 3 & 3 \\
\hline
\end{tabular}

We evaluated the performance of models performance at learning syntagmatic relations by computing the correlation of the ranking matrices obtained from semantic models with the key ranking. The higher the correlation score, the better the semantic model performed in binding different semantic relations to form new relations.

\subsection{Results}

\subsubsection{Paradigmatic Relatedness}

In the paradigmatic relatedness test, both spatial and graphical models were able to distinguish the nouns within categories from nouns outside categories. For both models, word relatedness was collapsed for within or across noun categories (animal, fruit, drink). The spatial model had an average within category relatedness of 0.41 ( $\mathrm{SE}=$ $0.09)$, and between category relatedness of $0.05(\mathrm{SE}=0.005)$. The graphical model had a within category relatedness score of $0.11(\mathrm{SE}=0.02)$, and a between categories relatedness score of $0.05(\mathrm{SE}=0.003)$.

\footnotetext{
${ }^{2}$ In this article, we use $\operatorname{Re}(v e r b$, noun), while the other direction $\operatorname{Re}($ noun,verb) remaining for followingup studies.
} 


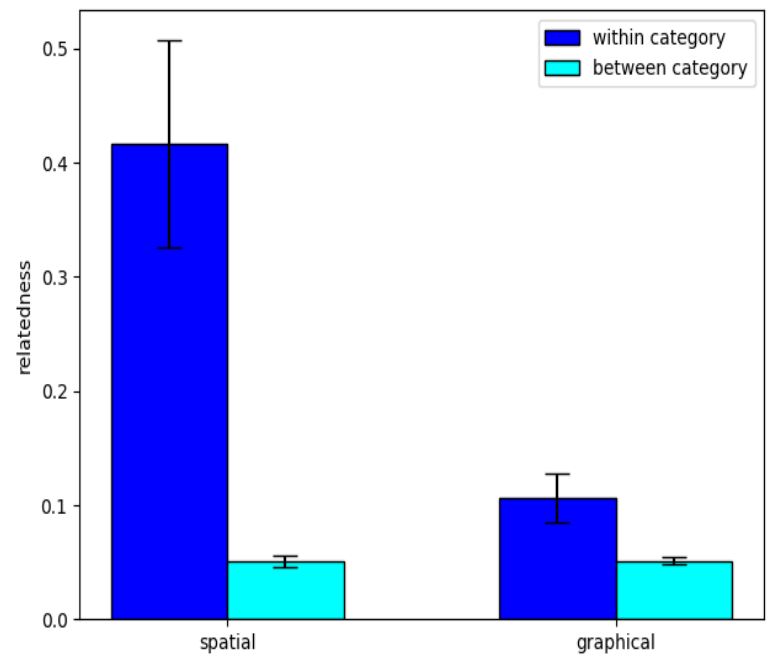

Figure 3: Mean paradigmatic Relatedness within vs. between noun categories

\subsubsection{Syntagmatic Relatedness}

In the syntagmatic relatedness test, the graphical model had an average correlation between its rankings and the key rankings of 0.96 ( $\mathrm{SE}=0.009$ ). The spatial model had an average correlation between its rankings and the key rankings of 0.67 (SE=0.005). We ran a mixed effect model testing the significance of this difference, with model type (spatial vs. graphical) and model ranking as independent variables predicting the key ranking as a dependent variable (with word-pair and simulation run as random factors). This model showed a significant difference interaction between model and rankings on the prediction of standard ranking, with $t=-13.19, p<2 \times 10^{-16}$. 


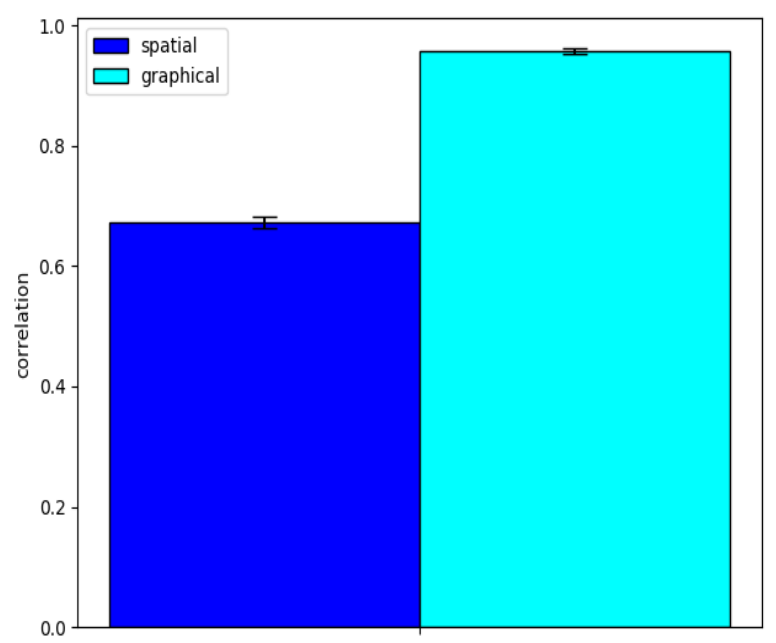

Figure 4: Mean correlation score of the ranking tasks

\section{Discussion}

Both the spatial models and graphical models demonstrated the capability to learn the semantic relatedness of paradigmatically-related words that belonged to the same category (according to the generative rules for the artificial world). These results are consistent with previous research demonstrating that spatial models are good at predicting semantic categories based on distributional information, and show that graphical models based on distributional information share this capacity (Huebner \& Willits, 2018; Landauer \& Dumais, 1997; M. N. Jones \& Mewhort, 2007).

However, the graphical model considerably outperformed the spatial model in terms of its ability to correctly predict syntagmatic relations. The spatial model showed little ability to take co-occurring noun-verb pairs like 'trap-rabbit', and paradigmaticallyrelated words like 'rabbit-deer', and bind them together to induce novel relations like 'trap-deer'. This failure could result from the failure of encoding syntagmatic relations and paradigmatic relations in the same semantic space. The failure could also be due in part to the nature of semantic relatedness measure in spatial model: spatial measures such as cosine similarity always calculate the relation between two words directly, and cannot combine two cosine scores to form 'indirect relations'. In order to do so, some kind of processing or combinatorial method is necessary, such as Kintsch's predication algorithm Kintsch (2001) or the spreading activation algorithm demonstrated in this paper.

Binding relations using a spreading activation method in graphical models is trivial: bridging 'trap' and 'deer' by 'rabbit', as rabbit is the intermediate unit that strongly relates to both words. The discrepancy in such relational tasks reflects the fundamental difference between two types of models in terms of representation: vector spaces are mathematical structures for representing features while graphs are a more explicit way to represent relations.

Similar to traditional distributional semantic models, the Distributional Graph Model 
developed in this article also encodes distributional information from a linguistic corpus, differentiating it from the previous semantic network models (along with Rotaru et al., 2018). Most previous models connect word nodes using association norms or $a$ priori defined relations like 'HAS A'. In contrast, the Distributional Graph Model connects word nodes using co-occurrence information in a linguistic corpus. Every time it takes in a sentence, the model effectively adds the sentence to the semantic network. By taking the advantage of linguistic corpus, training on the graphical model could be considered as a process of acquiring semantic knowledge and forming semantic memory by accumulating linguistic experience, rather than mere connection of word nodes.

Nevertheless, there are certain drawbacks of the Distributional Graph Model. For example, it is hard for this model, or graphical models in general, to encode ordered information across sentence boundaries, while most distributional semantic models can encode the ordered information using a moving window technique. The validity and the effectiveness of the semantic relatedness measure on graphical models developed in this article remains to be checked, regardless of its success in the particular semantic task in this study. Apart from the issues mentioned above, there is work to be done to extend the study described in the article, including comparison with other categories of distributional models (e.g. popular neural network models like Word2Vec, (Mikolov, Sutskever, Chen, Corrado, \& Dean, 2013)) and more experiments which check the underlying assumptions of the study.

There are multiple directions of future work concerning developing the model: 1) enlarging the artificial corpus and check the competence of the model in various semantic tasks (categorization, semantic similarity judgement); 2) applying the model in large scale naturalistic data (e.g. Wikipedia); 3) developing the model by adding constituent structure. One advantage of the distributional graph is that it can easily be altered to include hierarchical and syntactic structure information, in addition to simple word co-occurrences.

In the article, we develop a Distributional Graph Model, a semantic network model constructed from natural language corpus, which encodes distributional linguistic information. By representing distributional information in a graphical configuration, it outperforms its distributional precedents in binding syntagmatic and paradigmatic relations to form indirect semantic relations. By integrating distributional semantics into the graphical structure, this model utilizes the linguistic information in natural language corpus to construct the network, so that the formation of the semantic network becomes a statistical learning process, rather than a post hoc account for empirical data. Inheriting the advantages of both spatial distributional models and graphical models, the model we proposed here provide a representation of semantic memory with competence in representing indirect semantic relations, which is obtained by the acquisition of knowledge from linguistic experience.

\section{References}

Anderson, J. (1983). A spreading activation theory of memory. Journal of Verbal Learning and Verbal Behavior, 22(3), 261-295.

Asher, R. E., \& Simpson, J. M. Y. (1993). The encyclopedia of language and linguistics. Oxford: Pergamon.

Burgess, C., \& K.Lund. (2000). The dynamics of meaning in memory. Lawrence Erlbaum Associates. 
Collins, A., \& Loftus, E. (1975). A spreading-activation theory of semantic processing. Psychological Review, 82(6), 407-428.

Hjørland, B. (2015). Are relations in thesauri "context-free, definitional, and true in all possible worlds"? Journal of the Association for Information Science and Technology, 66(7), 1367-1373.

Huebner, P. A., \& Willits, J. A. (2018). Structured semantic knowledge can emerge automatically from predicting word sequences in child-directed speech. Frontiers in Psychology, 9, 133-151.

Jones, M., Willits, J., \& Dennis, S. (2014, 01). Models of semantic memory..

Jones, M. N., Kintsch, W., \& Mewhort, D. J. K. (2006). High-dimensional semantic space accounts of priming. Journal of Memory and Language, 55(4), 534-552.

Jones, M. N., \& Mewhort, D. J. (2007). Representing word meaning and order information in a composite holographic lexicon. Psychological Review, 114(1), 1-37.

Kintsch, W. (2001). Prediction. Cognitive Science, 25(2), 173-202.

Landauer, T., \& Dumais, S. (1997). A solution to plato's problem: The latent semantic analysis theory of acquisition, induction, and representation of knowledge. Psychological Review, 104(2), 211-240.

Lund, K., \& Burgess, C. (1996). Producing high-dimensional semantic spaces from lexical co-occurrence. Behavior Research Methods, Instruments, and Computers, 28(2), 203-208.

Mikolov, T., Sutskever, I., Chen, K., Corrado, G. S., \& Dean, J. (2013). Distributed representations of words and phrases and their compositionality. In In advances in neural information processing systems (pp. 3111-3119).

Rotaru, A. S., Vigliocco, G., \& Frank, S. L. (2018). Modeling the structure and dynamics of semantic processing. Cognitive Science, 42(8), 2890-2917. 